RESEARCH BRIEF

\title{
Trends in Hookah Use Among New York City Middle and High School Students, 2008-2014
}

Kristi Roods, $\mathrm{MPH}^{1}$; John Jasek, MPA${ }^{1}$; Shannon M. Farley, $\mathrm{DrPH}^{1}$

Accessible Version: www.cdc.gov/pcd/issues/2018/17_0283.htm

Suggested citation for this article: Roods K, Jasek J, Farley SM. Trends in Hookah Use Among New York City Middle and High School Students, 2008-2014. Prev Chronic Dis 2018;15:170283. DOI: https://doi.org/10.5888/pcd15.170283.

\section{PEER REVIEWED}

\section{Abstract}

We examined trends in hookah use among New York City middle and high school students. We calculated prevalences, linear trends, and odds ratios of ever and current hookah use, by selected demographic variables, using 2008 through 2014 data from the New York State Youth Tobacco Survey. The prevalence of ever hookah use increased overall from 2008 through 2014 (8.9\% to 13.0\%, $P$ $=.01$ ); current use was stable during this period but increased across many demographic characteristics. Our results indicate a need for efforts to educate populations with increasing prevalence of hookah use as well as policies that regulate use to reduce and denormalize hookah smoking.

\section{Objective}

Hookah use has increased among US adolescents and young adults (1-4). Attractiveness of flavored shisha, misperceptions about hazards posed by hookah use and types of shisha being served, and proliferation and limited regulation of hookah establishments contribute to this trend (5-8). Many hookah establishments claim to sell nontobacco shisha; however, research shows it is typically not tobacco-free (8). Even smoke from nontobacco shisha contains many toxic agents found in cigarette smoke (9). We examined trends in hookah use among New York City middle and high school students to better understand hookah use and guide policy.

\section{Methods}

The Youth Tobacco Survey (YTS) is a cross-sectional survey developed by the Centers for Disease Control and Prevention in conjunction with US states. YTS provides data on trends in youth tobacco use, access, and perceptions. New York State has conducted the survey biennially since 2000 and has included questions assessing hookah use since 2008. From 2008 through 2014 the survey was administered to a sample of 4,500 to 6,500 students in randomly selected New York City public and private school classrooms from grades 6 through 12. Participation rates (the school response rate multiplied by the student response rate) ranged from $70 \%$ to $83 \%$ during these survey years. All data were self-reported and were weighted to adjust for sex, race/ethnicity, and age (10).

Students were asked if they had ever used a hookah or waterpipe to smoke tobacco (ever hookah use) and if they had used a hookah or waterpipe to smoke tobacco at least one day in the past 30 days (current hookah use). Demographic variables assessed were school level (high school or middle school), sex (male or female), and race/ethnicity (Hispanic/Latino, white, black, or Asian). We computed prevalence of hookah use from 2008 through 2014 by demographic characteristics and by cigarette smoking status (never, former, or current). Former cigarette smoking was defined as having tried cigarettes but no use in the past 30 days. Current cigarette smoking was defined as having smoked at least once in the past 30 days. We tested for linear trend across years using $t$ tests (significance set at $P<.05$ ). We computed adjusted odds ratios (AORs) for ever and current hookah use among demographic and cigarette use variables. We used logistic regression models to compute AORs and corresponding $95 \%$ confidence intervals (CIs). Analyses were conducted by using SAS version 9.4 (SAS Institute, Inc). 


\section{Results}

The prevalence of ever hookah use increased overall from 2008 through $2014(8.9 \%$ to $13.0 \%, P=.01)$. It also increased among middle school students $(2.9 \%$ to $8.5 \%, P<.001)$, female students $(8.0 \%$ to $16.2 \%, P<.001)$, Hispanic or Latino students $(7.1 \%$ to $17.7 \%, P<.001)$, black students $(3.2 \%$ to $9.6 \%, P<.001)$, and former $(13.2 \%$ to $43.5 \%, P<.001)$ and never cigarette smokers $(2.1 \%$ to $6.2 \%, P<.001)$. In 2014 , Hispanic or Latino students had higher odds of ever using hookah than all other racial/ethnic groups. Female students ( $\mathrm{AOR}=2.6$; 95\% CI, 1.8-3.9) had higher odds of ever using hookah than male students. Former (AOR = $11.9 ; 95 \% \mathrm{CI}, 8.8-16.2)$ and current cigarette smokers $(\mathrm{AOR}=$ 22.3 ; 95\% CI, 13.0-38.5) had higher odds of ever use than never cigarette smokers. The odds of ever using hookah among middle school students (AOR $=0.7 ; 95 \%$ CI, 0.5-1.1) were not significantly different from odds of use among high school students (Table 1).

Current hookah use was flat overall $(4.8 \%$ to $5.5 \%, P=.33)$ but increased among middle school (1.6\% to $4.5 \%, P<.001)$, female (3.8\% to $6.9 \%, P=.005)$, Hispanic or Latino $(3.6 \%$ to $8.3 \%, P<$ $.001)$, and black students $(2.0 \%$ to $3.5 \%, P=.04)$. It decreased among white $(10.9 \%$ to $5.2 \%, P=.01)$ and Asian students $(5.6 \%$ to $2.4 \%, P=.049)$. The prevalence also increased among former $(4.8 \%$ to $14.3 \%, P=.002)$ and never cigarette smokers $(0.7 \%$ to $2.3 \%, P<.001$ ). In 2014, female students (AOR $=2.5 ; 95 \% \mathrm{CI}$, 1.4-4.5) had higher odds of current hookah use than male students. Former (AOR = 7.1; 95\% CI, 3.6-14.0) and current cigarette smokers $(\mathrm{AOR}=30.9 ; 95 \% \mathrm{CI}, 19.0-50.3)$ had higher odds than never cigarette smokers. The odds were significantly lower among black $(\mathrm{AOR}=0.47 ; 95 \% \mathrm{CI}, 0.3-0.8)$ and Asian $(\mathrm{AOR}=$ $0.4 ; 95 \%$ CI, 0.2-0.8) students than among Hispanic or Latino students; odds of hookah use between white students and Hispanic or Latino students was not significant (AOR $=0.6$; 95\% CI, 0.3-1.2). We found no significant difference in odds between middle (AOR $=1.2 ; 95 \%$ CI, 0.6-2.5) and high school students (Table 2).

\section{Discussion}

Our study results show that ever and current hookah use among middle school, female, and Latino and black students increased over time, while current use among white and Asian students decreased. The most disconcerting changes were among female and Hispanic or Latino students, among whom use increased significantly since 2008 . These data differ from those of earlier studies of students from 2005 through $2008(1,2)$, which showed more prevalent hookah use among white and male students, and studies nationally and statewide from 2010 through $2012(3,4)$, which showed closing gaps in the prevalence of hookah use between Latino and white students and between male and female students.

Hookah use is increasing among never and former cigarette smokers. Focus groups among urban young adults demonstrated extensive awareness of alternative tobacco products but disagreement about associated harms (7). Another study showed that hookah use among non-cigarette smokers predicted cigarette smoking initiation, current cigarette smoking, and higher intensity of cigarette smoking at 2-year follow-up, suggesting that hookah use is a precursor to cigarette smoking (11).

Limitations to our study include that YTS is cross-sectional, provides only population snapshots, and collects self-reported data; therefore, results are subject to bias. However, because YTS has collected data on hookah use from both private and public schools since 2008, it is the best available data source and is representative of New York City students.

Our results indicate that hookah use among middle school and high school students in New York City is proliferating, and efforts should be made to educate populations with increasing prevalence. Policies that limit or regulate hookah use, such as increasing taxes to create parity with other tobacco products and extending the city's smoking ban to regulate nontobacco shisha in public establishments to limit access and denormalize the practice, are suggested. These policies have been implemented across jurisdictions nationally (12). Jurisdictions considering cigarette-specific legislation should also monitor changes in alternative products such as hookah and e-cigarettes.

\section{Acknowledgments}

We have no sources of financial support to report.

\section{Author Information}

Corresponding Author: Kristi Roods, MPH, New York City Department of Health and Mental Hygiene, 42-09 28th St, WS 11 157, Long Island City, NY 11101. Telephone: 347-396-4603. Email: kmcconnell@health.nyc.gov.

Author Affiliations: ${ }^{1}$ New York City Department of Health and Mental Hygiene, Long Island City, New York.

\footnotetext{
The opinions expressed by authors contributing to this journal do not necessarily reflect the opinions of the U.S. Department of Health and Human Services, the Public Health Service, the Centers for Disease Control and Prevention, or the authors' affiliated institutions.
} 


\section{References}

1. Primack BA, Walsh M, Bryce C, Eissenberg T. Water-pipe tobacco smoking among middle and high school students in Arizona. Pediatrics 2009;123(2):e282-8.

2. Smith JR, Edland SD, Novotny TE, Hofstetter CR, White MM, Lindsay SP, et al. Increasing hookah use in California. Am J Public Health 2011;101(10):1876-9.

3. Barnett TE, Forrest JR, Porter L, Curbow BA. A multiyear assessment of hookah use prevalence among Florida high school students. Nicotine Tob Res 2014;16(3):373-7.

4. Singh T, Arrazola RA, Corey CG, Husten CG, Neff LJ, Homa DM, et al. Tobacco use among middle and high school students - United States, 2011-2015. MMWR Morb Mortal Wkly Rep 2016;65(14):361-7.

5. Ambrose BK, Day HR, Rostron B, Conway KP, Borek N, Hyland A, et al. Flavored tobacco product use among US youth aged 12-17 years, 2013-2014. JAMA 2015; 314(17):1871-3.

6. Cawkwell PB, Lee L, Weitzman M, Sherman SE. Tracking hookah bars in New York: utilizing yelp as a powerful public health tool. JMIR Public Health Surveill 2015;1(2).

7. Wray RJ, Jupka K, Berman S, Zellin S, Vijaykumar S. Young adults' perceptions about established and emerging tobacco products: results from eight focus groups. Nicotine Tob Res 2012;14(2):184-90.

8. Zhou S, Weitzman M, Vilcassim R, Wilson J, Legrand N, Saunders E, et al. Air quality in New York City hookah bars. Tob Control 2015;24(e3):e193-8.

9. Hammal F, Chappell A, Wild TC, Kindzierski W, Shihadeh A, Vanderhoek A, et al. 'Herbal' but potentially hazardous: an analysis of the constituents and smoke emissions of tobaccofree waterpipe products and the air quality in the cafés where they are served. Tob Control 2015;24(3):290-7.

10. New York Youth Tobacco Survey overview. Albany (NY): New York State Department of Health; 2014. https:// health.data.ny.gov/Health/Youth-Tobacco-Survey-Beginning2000/pbq7-ddg9. Accessed December 15, 2016.

11. Soneji S, Sargent JD, Tanski SE, Primack BA. Associations between initial water pipe tobacco smoking and snus use and subsequent cigarette smoking: results from a longitudinal study of US adolescents and young adults. JAMA Pediatr 2015; 169(2):129-36.

12. Legal Resource Center for Public Health Policy. 50 State survey of hookah regulations. Baltimore (MD): University of Maryland Francis King Carey School of Law; 2013. https:// www.law.umaryland.edu/programs/publichealth/documents/ Hookah_50_State_Survey.pdf. Accessed January 11, 2017.

The opinions expressed by authors contributing to this journal do not necessarily reflect the opinions of the U.S. Department of Health and Human Services, the Public Health Service, the Centers for Disease Control and Prevention, or the authors' affiliated institutions. 


\section{Tables}

Table 1. Prevalence and Adjusted Odds of Ever Hookah Use Among New York City Middle and High School Students, by Selected Demographic Characteristics, New York Youth Tobacco Survey 2008, 2010, 2012, and 2014

\begin{tabular}{|c|c|c|c|c|c|c|c|c|c|c|}
\hline \multirow[b]{2}{*}{ Characteristic } & \multicolumn{2}{|r|}{2008} & \multicolumn{2}{|r|}{2010} & \multicolumn{2}{|r|}{2012} & \multicolumn{3}{|c|}{2014} & \multirow{2}{*}{$\begin{array}{c}P \\
\text { Value }\end{array}$} \\
\hline & No. ${ }^{a}$ & $\%(95 \% \mathrm{Cl})$ & No. $^{a}$ & $\%(95 \% \mathrm{Cl})$ & No. $^{a}$ & $\%(95 \% \mathrm{Cl})$ & No. $^{a}$ & $\%(95 \% \mathrm{Cl})$ & AOR $^{b}(95 \% \mathrm{Cl})$ & \\
\hline \multicolumn{11}{|l|}{ School level } \\
\hline Overall & 57,000 & $8.9(5.9-12.0)$ & 61,000 & $11.1(8.4-13.8)$ & 82,000 & $13.8(10.8-16.7)$ & 72,000 & $13.0(10.8-15.2)$ & - & .01 \\
\hline \multicolumn{11}{|l|}{ School level } \\
\hline High school & 47,000 & $13.1(8.3-17.9)$ & 50,000 & $15.6(11.6-19.5)$ & 62,000 & $18(14.1-21.9)$ & 51,000 & $16.1(13.4-18.9)$ & $1.00[$ Ref $]$ & .19 \\
\hline Middle school & 8,000 & $2.9(1.9-4.0)$ & 10,000 & $4.4(3.3-5.6)$ & 19,000 & $7.8(6.2-9.3)$ & 20,000 & $8.5(6.7-10.3)$ & $0.72(0.5-1.1)$ & $<.001$ \\
\hline \multicolumn{11}{|l|}{ Sex } \\
\hline Male & 32,000 & $9.8(6.2-13.5)$ & 30,000 & $11.1(8.0-14.2)$ & 41,000 & $13.5(10.3-16.6)$ & 22,000 & $9.2(7.0-11.4)$ & $1.00[$ Ref] & .95 \\
\hline Female & 25,000 & $8.0(4.5-11.5)$ & 32,000 & $11.2(8.3-14.0)$ & 41,000 & $14.1(10.3-17.9)$ & 50,000 & $16.2(13.4-18.9)$ & $2.62(1.8-3.9)$ & $<.001$ \\
\hline \multicolumn{11}{|l|}{ Race/ethnicity } \\
\hline Hispanic/Latino & 16,000 & $7.1(4.5-9.7)$ & 24,000 & $11.9(8.5-15.3)$ & 40,000 & $18.4(14.5-22.3)$ & 37,000 & $17.7(13.7-21.8)$ & $1.00[$ Ref $]$ & $<.001$ \\
\hline White & 25,000 & $20.3(13.1-27.6)$ & 22,000 & $19.6(12.5-26.7)$ & 22,000 & $18.9(12.4-25.4)$ & 10,000 & $11.8(7.4-16.1)$ & $0.62(0.4-0.9)$ & .06 \\
\hline Black & 6,000 & $3.2(1.6-4.7)$ & 6,000 & $4.0(2.1-5.9)$ & 9,000 & $5.9(3.9-7.9)$ & 15,000 & $9.6(7.7-11.5)$ & $0.60(0.4-0.8)$ & $<.001$ \\
\hline Asian & 6,000 & $10.6(6.4-14.7)$ & 6,000 & $9.6(7.0-12.3)$ & 6,000 & $9.5(6.5-12.5)$ & 5,000 & $7.2(4.6-9.8)$ & $0.39(0.2-0.7)$ & .19 \\
\hline \multicolumn{11}{|c|}{ Cigarette smoking status } \\
\hline Never & 9,000 & $2.1(1.5-2.7)$ & 14,000 & $3.5(2.5-4.5)$ & 24,000 & $5.3(3.9-6.8)$ & 28,000 & $6.2(4.7-7.8)$ & $1.00[$ Ref $]$ & $<.001$ \\
\hline Former & 15,000 & $13.2(10.1-16.3)$ & 23,000 & $27.7(21.9-33.6)$ & 23,000 & $28.5(23.6-33.4)$ & 24,000 & $43.5(37.0-50.0)$ & $11.92(8.8-16.2)$ & $<.001$ \\
\hline Current & 24,000 & $40.3(36.4-44.2)$ & 17,000 & $51.7(42.2-61.1)$ & 21,000 & $66.9(58.6-75.1)$ & 10,000 & $60.6(47.9-73.2)$ & $22.35(13.0-38.5)$ & .05 \\
\hline
\end{tabular}

Abbreviations: - , does not apply; AOR, adjusted odds ratio; $\mathrm{Cl}$, confidence interval; Ref, reference.

${ }^{a}$ Weighted $\mathrm{n}$ is rounded to the nearest thousand.

${ }^{\mathrm{b}}$ AORs calculated by using a logistic regression model, adjusting for school level, sex, race/ethnicity, and cigarette use.

${ }^{\mathrm{c}} P$ value for linear trend calculated by using $t$ test; significance set at $P<.05$. 
Table 2. Prevalence and Adjusted Odds of Current Hookah Use Among New York City Middle and High School Students, by Selected Demographic Characteristics, New York Youth Tobacco Survey 2008, 2010, 2012, and 2014

\begin{tabular}{|c|c|c|c|c|c|c|c|c|c|c|}
\hline \multirow[b]{2}{*}{ Characteristic } & \multicolumn{2}{|c|}{2008} & \multicolumn{2}{|c|}{2010} & \multicolumn{2}{|c|}{2012} & \multicolumn{3}{|c|}{2014} & \multirow{2}{*}{$\stackrel{P}{\text { Value }^{c}}$} \\
\hline & No. $^{a}$ & $\%(95 \% \mathrm{Cl})$ & No. $^{a}$ & $\%(95 \% \mathrm{Cl})$ & No. $^{a}$ & $\%(95 \% \mathrm{Cl})$ & No. $^{a}$ & $\%(95 \% \mathrm{Cl})$ & $\mathrm{AOR}^{\mathrm{b}}(95 \% \mathrm{Cl})$ & \\
\hline Overall & 30,000 & $4.8(3.2-6.5)$ & 30,000 & $5.5(3.9-7.1)$ & 38,000 & $6.5(5.0-8.0)$ & 30,000 & $5.5(4.4-6.7)$ & - & .33 \\
\hline
\end{tabular}

School level

\begin{tabular}{|c|c|c|c|c|c|c|c|c|c|c|}
\hline High school & 25,000 & $7.0(4.6-9.4)$ & 25,000 & $7.7(5.4-10.1)$ & 28,000 & $8.1(5.9-10.3)$ & 20,000 & $6.3(4.5-8.0)$ & $1.00[$ Ref $]$ & .70 \\
\hline Middle school & 4,000 & $1.6(0.9-2.4)$ & 5,000 & $2.2(1.3-3.0)$ & 10,000 & $4.1(3.0-5.1)$ & 10,000 & $4.5(3.3-5.8)$ & $1.23(0.6-2.5)$ & $<.001$ \\
\hline
\end{tabular}

Sex

\begin{tabular}{|c|c|c|c|c|c|c|c|c|c|c|}
\hline Male & 18,000 & $5.8(3.6-8.0)$ & 15,000 & $5.5(3.8-7.3)$ & 20,000 & $6.6(5.0-8.2)$ & 9,000 & $3.9(2.6-5.2)$ & $1.00[$ Ref $]$ & .24 \\
\hline Female & 12,000 & $3.8(2.4-5.3)$ & 15,000 & $5.5(3.8-7.1)$ & 18,000 & $6.4(4.4-8.3)$ & 21,000 & $6.9(5.2-8.5)$ & $2.50(1.4-4.5)$ & .005 \\
\hline
\end{tabular}

Race/ethnicity

\begin{tabular}{|c|c|c|c|c|c|c|c|c|c|c|}
\hline Hispanic/Latino & 8,000 & $3.6(2.0-5.2)$ & 13,000 & $6.6(3.9-9.2)$ & 20,000 & $9.3(7.0-11.6)$ & 17,000 & $8.3(6.4-10.1)$ & $1.00[$ Ref $]$ & $<.001$ \\
\hline White & 14,000 & $10.9(7.3-14.6)$ & 11,000 & $10.2(6.3-14.0)$ & 10,000 & $8.3(4.6-12.0)$ & 4,000 & $5.2(2.5-7.9)$ & $0.60(0.3-1.2)$ & .01 \\
\hline Black & 3,000 & $2.0(1.2-2.9)$ & 2,000 & $1.1(0.3-1.8)$ & 3,000 & $2.1(0.9-3.4)$ & 5,000 & $3.5(2.0-4.9)$ & $0.47(0.3-0.8)$ & .04 \\
\hline Asian & 3,000 & $5.6(2.8-8.3)$ & 3,000 & $4.4(2.7-6.0)$ & 3,000 & $4.0(1.9-6.1)$ & 1,000 & $2.4(0.8-4.0)$ & $0.41(0.2-0.8)$ & .049 \\
\hline
\end{tabular}

Cigarette smoking status

\begin{tabular}{|c|c|c|c|c|c|c|c|c|c|c|}
\hline Never & 3,000 & $0.7(0.2-1.1)$ & 6,000 & $1.4(0.8-2.0)$ & 9,000 & $2.0(1.2-2.8)$ & 10,000 & $2.3(1.5-3.1)$ & $1.00[$ Ref $]$ & $<.001$ \\
\hline Former & 5,000 & $4.8(3.3-6.2)$ & 9,000 & $10.8(7.0-14.6)$ & 7,000 & $9.1(6.0-12.2)$ & 8,000 & $14.3(9.1-19.5)$ & $7.11(3.6-14.0)$ & .002 \\
\hline Current & 17,000 & $34.4(28.4-40.4)$ & 11,000 & $34.9(26.0-43.8)$ & 12,000 & $38.6(26.7-47.6)$ & 6,000 & $40.9(33.1-48.7)$ & $30.9(19.0-50.3)$ & .15 \\
\hline
\end{tabular}

Abbreviations: - , does not apply; AOR, adjusted odds ratio; $\mathrm{Cl}$, confidence interval; Ref, reference.

${ }^{a}$ Weighted $\mathrm{n}$ is rounded to the nearest thousand.

${ }^{\mathrm{b}}$ AORs calculated by using a logistic regression model adjusting for school level, sex, race/ethnicity, and cigarette use.

${ }^{\mathrm{C}} P$ value for linear trend calculated by using $t$ test; significance set at $P<.05$. 